\title{
Quantum Forms of Tensor Products
}

\author{
Daniel Kastler and Daniel Testard \\ Centre de Physique Théorique, Luminy Case 907, F-13288 Marseille Cedex 9 \\ and Université d'Aix-Marseille II, France
}

Received July 24, 1992; in revised form November 24, 1992

Dedicated to Huzihiro Araki

Abstract. We describe the skew tensor-product structure inherent to Yang-Mills
procedures pertaining to non-commutative spaces obtained by (skew) tensorization.

\section{Introduction}

In the fall of 1991 Alain Connes introduced [2] the differential algebra of quantum differential forms (non-commutative generalization of the classical De Rham complex) and used this concept to obtain an improved form of his non-commutative Yang-Mills scheme, in particular as applied to the standard model of elementary particles. The improvement resides in the removal of the cumbersome adynamical fields which plagued the previous formalism (a price to be paid for the use of formal differential forms instead of the genuine quantum differential forms). We refer our reader to ref. [2] versus ref. [1] - or, for more details, to [3] III versus [3] I and II, for a comparison of the two methods.

Let $\mathbf{A}$ be a (generally non-commutative, possibly $\mathbf{Z} / 2$-graded) associative *-algebra endowed with the (non-commutative) riemannian geometry specified by a $D$-summable $K$-cycle $(H, D)$ [2]. The generalized De Rham complex (or set of $D$-quantum forms) $\Omega_{D} \mathbf{A}$ of $\mathbf{A}$ if the differential algebra, with differential $\delta$, quotient of the unital differential envelope $(\Omega \mathbf{A}, \delta)$ of $\mathbf{A}$ (cf. e.g. [4]) by the graded ideal $K^{*}+\delta K^{*}, K^{*}=\bigoplus_{n \in \mathbf{N}} \operatorname{Ker} \pi_{D} \cap \Omega \mathbf{A}^{n}$, homogenized kernel of the representation $\pi_{D}$ of $\Omega \mathbf{A}$ specified by $D$ (cf. [2]).

In the application to the standard model, the (electro-weak side of) the algebra is the tensor product $\mathbf{A}=C^{\infty}(\mathbf{M}) \otimes(\mathbf{C} \oplus \mathbf{H})$ of the algebra of smooth functions on the space-time $\operatorname{spin}^{c}$ riemannian manifold $\mathbf{M}$ by the algebra $\mathbf{C} \oplus \mathbf{H}$ embodying the $U(1) \times S U(2)$ (innerspace-) degrees of freedom. The $K$-cycle $D$ is the tensor product (see below (16)) of the Dirac $K$-cycle of $C^{\infty}(\mathbf{M})$ by an inner-space $K$-cycle $\left(H_{f}, D_{f}\right)$ specified by the mass matrix of the fermions. This tensor product structure raises the question of how the differential algebra $\Omega_{D} \mathbf{A}$ of electro-weak quantum forms relates 
to the sets $\Omega_{D} C^{\infty}(\mathbf{M}) \cong \Omega(\mathbf{M})$ and $\Omega_{D_{f}}(\mathbf{C} \otimes \mathbf{H})$ of usual differential forms and of "inner-space quantum forms". In this note we will partially answer this question by exhibiting skew tensor-product structures which shed light on the computation of the non-commutative Yand-Mills action (cf. [3] III).

Placing ourselves in the general context (useful in supersymmetric theories) of $\mathbf{Z} / 2$ graded real (or complex) algebras, we more generally look at the non-commutative De Rham complex $\Omega_{D} \mathbf{A}$ for a *-algebra skew tensor product $\mathbf{A}=\mathbf{A}^{\prime} \hat{\otimes} \mathbf{A}^{\prime \prime}$ of two *-algebras $\mathbf{A}^{\prime}$ and $\mathbf{A}^{\prime \prime}$ respectively equipped with $K$-cycles $\left(H^{\prime}, D^{\prime}, \chi^{\prime}\right)$ and $\left(H^{\prime \prime}, D^{\prime \prime}, \chi^{\prime \prime}\right)$, the $K$-cycle over A being the tensor-product $\left.(H, D, \chi)=H^{\prime}, D^{\prime}, \chi^{\prime}\right) \otimes$ $\left(H^{\prime \prime}, D^{\prime}, \chi^{\prime \prime}\right)$ (see below (16)).

At the level of unital differential envelopes, treated in Sect.I, the situation is quite simple: the unital differential envelope of the skew tensor-product maps homomorphically onto the skew tensor product of the differential envelopes of the factor algebras. At the level of the representation, we have the skew tensor-product $\pi_{D}=\pi_{D^{\prime}} \hat{\otimes} \pi_{D^{\prime \prime}}$ as shown in Sect. II. The state of affairs is less transparent at the level of the non-commutative De Rham complex $\Omega_{D} \mathbf{A}$, since the tensor products $\Omega \mathbf{A}^{\prime r} \hat{\otimes} \Omega \mathbf{A}^{\prime \prime s}$ and $\Omega \mathbf{A}^{\prime p} \hat{\otimes} \Omega \mathbf{A}^{\prime \prime q}$ for $r+s=p+q \bmod 2$ intersect each other nontrivially in the $\pi_{D}$ representation. In lack of general statements, we discuss the levels relevant to the Yang-Mills calculations, where a tensor-product structure still holds for the set $\pi_{D}\left(\delta K^{1}\right)$ by which one has to divide $\pi_{D}\left(\Omega \mathbf{A}^{2}\right)$ in order to get the module $\Omega_{D} \mathbf{A}^{2}$ of two-quantum-forms.

\section{Differential Envelopes of Tensors Products}

Let $\mathbf{A}=\mathbf{A}^{0} \oplus \mathbf{A}^{1}$ be a unital $\mathbf{Z} / 2$-graded real (or complex) *-algebra. We recall that the unital differential envelope $\Omega \mathbf{A}$ of $\mathbf{A}$ (a bigraded differential algebra) ${ }^{1}$, whose $\mathbf{Z} / 2$-grading proceeds from its total grading, with differential $\delta$, containing $\mathbf{A}$ as a $\mathbf{Z} / 2$-graded subalgebra, and linearly generated by elements of the form:

$$
a_{0} \delta a_{1} \ldots \delta a_{n}, \quad a_{0}, a_{1}, \ldots, a_{n} \in \mathbf{A},
$$

can be specified by the following universality property: given a unital real (or complex) $\mathbf{Z} / 2$-graded differential algebra $\left(\Xi=\Xi^{0} \oplus \Xi^{1}, \Delta\right)$ and a zero-grade unital homomorphism $\phi: \mathbf{A} \rightarrow \Xi$ of $\mathbf{Z} / 2$-graded algebras, $\phi$ extends uniquely to a homomorphism $\bar{\phi}: \Omega \mathbf{A} \rightarrow \Xi$ of bigraded differential algebras:

$\Omega \mathbf{A}$

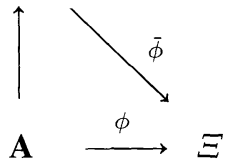

(specifically given by:

$$
\left.\bar{\phi}\left(a_{0} \delta a_{1} \ldots \delta a_{n}\right)=\left(\phi a_{0}\right)\left(\Delta \phi a_{1}\right) \ldots\left(\Delta \phi a_{n}\right), \quad a_{0}, a_{1}, \ldots, a_{n} \in \mathbf{A}\right) .
$$

I.1 Proposition. Let $\mathbf{A}^{\prime}=\mathbf{A}^{\prime 0} \oplus \mathbf{A}^{\prime 1}$ and $\mathbf{A}^{\prime \prime}=\mathbf{A}^{\prime \prime 0} \oplus \mathbf{A}^{\prime \prime 1}$ be unital $\mathbf{Z} / 2$-graded (real or complex) *-algebras, with respective unital differential envelopes $\left(\Omega \mathbf{A}^{\prime}, \delta^{\prime}\right)$ and

\footnotetext{
1 For homogeneous elements $a_{0}, a_{1}, \ldots, a_{n} \in \mathbf{A}$, the element (1) of $\Omega \mathbf{A}$ has $\mathbf{N}$-grade $n$ and total grade $n+\partial a_{1}+\ldots+\partial a_{n}$, denoting by $\partial a$ the grade of the homogeneous element $a \in \mathbf{A}$. We denote by $\Omega \mathbf{A}^{n}$ the set of elements $\Omega \mathbf{A}$ of $\mathbf{N}$-grade $n$
} 
$\left(\Omega \mathbf{A}^{\prime \prime}, \delta^{\prime \prime}\right)$. Let $\mathbf{A}=\mathbf{A}^{\prime} \hat{\otimes} \mathbf{A}^{\prime \prime}$ be the skew tensor product of $\mathbf{A}^{\prime}$ and $\mathbf{A}^{\prime \prime}$ with differential envelope $(\Omega \mathbf{A}, \delta)$. Set $\Omega \mathbf{A}_{\otimes}=\Omega \mathbf{A}^{\prime} \hat{\otimes} \Omega \mathbf{A}^{\prime \prime}$, with differential $\delta_{\otimes}$, for the skew product of $\left(\Omega \mathbf{A}^{\prime}, \delta^{\prime}\right)$ and $\left(\Omega \mathbf{A}^{\prime \prime}, \delta^{\prime \prime}\right)$ :

$$
\left\{\begin{array}{l}
\Omega \mathbf{A}_{\otimes}^{n}=\sum_{p+q=n} \Omega \mathbf{A}^{\prime p} \hat{\otimes} \Omega \mathbf{A}^{\prime \prime q}, \\
\delta_{\otimes}\left(a^{\prime} \otimes a^{\prime \prime}\right)=\delta^{\prime} a^{\prime} \hat{\otimes} a^{\prime \prime}+(-1)^{\partial a^{\prime}} a^{\prime} \hat{\otimes} \delta^{\prime \prime} a^{\prime \prime}, \quad a^{\prime} \in \mathbf{A}^{\prime}, a^{\prime \prime} \in \mathbf{A}^{\prime \prime} .
\end{array}\right.
$$

Then $\Omega \mathbf{A}$ maps homomorphically onto $\Omega \mathbf{A}_{\otimes}$ as bigraded differential algebras.

Specifically the homomorphism $\bar{i}: \Omega \mathbf{A} \rightarrow \Omega \mathbf{A}_{\otimes}$ is obtained as follows by universality of $\Omega \mathbf{A}$ :

\section{$\Omega \mathbf{A}$}

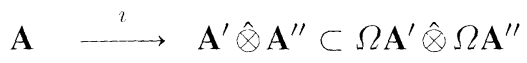

i.e. one has, for $a_{0}^{\prime}, a_{1}^{\prime}, \ldots, a_{n}^{\prime} \in \mathbf{A}$, of respective grades $\partial^{\prime} a_{0}^{\prime}, \partial^{\prime} a_{1}^{\prime}, \ldots, \partial^{\prime} a^{\prime} n$, and $a_{0}^{\prime \prime}, a_{1}^{\prime \prime}, \ldots, a_{n}^{\prime \prime} \in \mathbf{A}^{\prime \prime} .{ }^{2}$

$$
\begin{aligned}
\bar{i}\left[\left(a_{0}^{\prime} \hat{\otimes} a_{0}^{\prime \prime}\right) \delta\left(a_{1}^{\prime} \hat{\otimes} a_{1}^{\prime \prime}\right) \ldots \delta\left(a_{n}^{\prime} \hat{\otimes} a_{n}^{\prime \prime}\right)\right]= & \left(a_{0}^{\prime} \hat{\otimes} a_{0}^{\prime \prime}\right)\left[\delta^{\prime} a_{1}^{\prime} \hat{\otimes} a_{1}^{\prime \prime}+(-1)^{\partial a_{1}^{\prime}} a_{1}^{\prime} \hat{\otimes} \delta a_{1}^{\prime \prime}\right] \\
& \ldots\left[\delta^{\prime} a_{n}^{\prime} \hat{\otimes} a_{n}^{\prime \prime}+(-1)^{\partial a_{n}^{\prime}} a_{n}^{\prime} \hat{\otimes} \delta a_{n}^{\prime \prime}\right] .
\end{aligned}
$$

This map $\bar{i}$ is linear, multiplicative, preserves the intrinsic grade and the $N$-grade, intertwines the differentials ${ }^{3} \delta$ and $\delta_{\otimes}$ :

$$
\bar{i} \circ \delta=\delta_{\otimes} \circ \bar{i}
$$

and has a right inverse $J$ given as follows: one has for $\omega^{\prime} \in \Omega \mathbf{A}^{\prime}$ and $\omega^{\prime \prime} \in \Omega \mathbf{A}^{\prime \prime}$ :

$$
J\left(\omega^{\prime} \hat{\otimes} \omega^{\prime \prime}\right)=\bar{j}^{\prime}\left(\omega^{\prime}\right) \bar{j}^{\prime \prime}\left(\omega^{\prime \prime}\right),
$$

where the maps $\bar{j}^{\prime}: \Omega \mathbf{A}^{\prime} \rightarrow \Omega \mathbf{A}$ and $\bar{j}^{\prime \prime}: \Omega \mathbf{A}^{\prime \prime} \rightarrow \Omega \mathbf{A}$ are associated by the universality diagrams

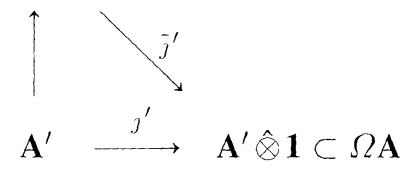

resp.:

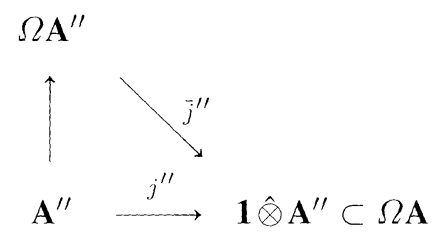

\footnotetext{
2 Tensor products $\hat{\otimes}$ of normal forms are just ordinary tensor products $\otimes$ : the notation $\hat{\otimes}$ recalls the skew multiplication rule of $\Omega \mathbf{A}^{\prime} \hat{\otimes} \Omega \mathbf{A}^{\prime \prime}$

$3 \delta$ and $\delta_{\otimes}$ are both graded derivations of intrinsic grade zero and $N$-grade one with vanishing square
} 
to the respective canonical injections $j^{\prime}: \mathbf{A}^{\prime} \rightarrow \mathbf{A}^{\prime} \hat{\otimes} \mathbf{1} \subset \Omega \mathbf{A}$ and $j^{\prime \prime}: \mathbf{A}^{\prime \prime} \rightarrow \mathbf{1} \hat{\otimes} \mathbf{A}^{\prime \prime} \subset$ $\Omega \mathbf{A}$ : in other terms one has for $a_{0}^{\prime}, a_{1}^{\prime}, \ldots, a_{n}^{\prime} \in \mathbf{A}^{\prime}$

$$
\bar{j}^{\prime}\left(a_{0}^{\prime} \delta^{\prime} a_{1}^{\prime} \ldots \delta^{\prime} a_{n}^{\prime}\right)=\left(a_{0}^{\prime} \hat{\otimes} \mathbf{1}\right) \delta\left(a_{1}^{\prime} \hat{\otimes} \mathbf{1}\right) \ldots \delta\left(a_{n}^{\prime} \hat{\otimes} \mathbf{1}\right),
$$

and for $a_{0}^{\prime \prime}, a_{1}^{\prime \prime}, \ldots, a_{n}^{\prime \prime} \in \mathbf{A}^{\prime \prime}$,

$$
\bar{j}^{\prime \prime}\left(a_{0}^{\prime \prime} \delta^{\prime \prime} a_{1}^{\prime \prime} \ldots \delta^{\prime \prime} a_{n}^{\prime \prime}\right)=\left(\mathbf{1} \hat{\otimes} a_{0}^{\prime \prime}\right) \delta\left(\mathbf{1} \hat{\otimes} a_{1}^{\prime \prime}\right) \ldots \delta\left(\mathbf{1} \hat{\otimes} a_{n}^{\prime \prime}\right) .
$$

In fact one has:

$$
\begin{cases}\bar{i} \circ \bar{j}^{\prime}\left(\omega^{\prime}\right)=\omega^{\prime} \hat{\otimes} \mathbf{1}, & \omega^{\prime} \in \Omega \mathbf{A}^{\prime}, \\ \bar{i} \circ \bar{j}^{\prime \prime}\left(\omega^{\prime \prime}\right)=\mathbf{1} \hat{\otimes} \omega^{\prime \prime}, & \omega^{\prime \prime} \in \Omega \mathbf{A}^{\prime \prime} .\end{cases}
$$

As a consequence the kernel $\mathbf{k}$ of $\bar{i}$ is a bigraded differential ideal of $\Omega \mathbf{A}$, with

$$
\Omega \mathbf{A}_{\otimes}^{\prime \prime}=\Omega \mathbf{A}^{n} / \mathbf{k}^{n}
$$

where $\mathbf{k}^{n}=\Omega \mathbf{A}^{n} \cap \mathbf{k}$.

Proof. All claims obviously follow from the definitions but the relations (9) and the right inverse property of $J$.

Check of (9): The maps $\bar{i}, \bar{j}^{\prime}$, and $\bar{j}^{\prime \prime}$ are homomorphisms of $\mathbf{N}$-graded differential algebras by construction. We have, from (4a) and (6a), for $\omega^{\prime}=a_{0}^{\prime} \delta^{\prime} a_{1}^{\prime} \ldots \delta^{\prime} a_{n}^{\prime}$ :

$$
\begin{aligned}
\bar{i} \circ \bar{j}^{\prime}\left(\omega^{\prime}\right) & =\bar{i}\left[\left(a_{0}^{\prime} \hat{\otimes} \mathbf{1}\right) \delta\left(a_{1}^{\prime} \hat{\otimes} \mathbf{1}\right) \ldots \delta\left(a_{n}^{\prime} \hat{\otimes} \mathbf{1}\right)\right] \\
& =\left(a_{0}^{\prime} \hat{\otimes} \mathbf{1}\right)\left(\delta^{\prime} a_{1}^{\prime} \hat{\otimes} \mathbf{1}\right) \ldots\left(\delta^{\prime} a_{n}^{\prime} \hat{\otimes} \mathbf{1}\right)=\omega^{\prime} \hat{\otimes} \mathbf{1} .
\end{aligned}
$$

and, analogously, from (4a) and (7a), for $\omega^{\prime \prime}=a_{0}^{\prime \prime} \delta^{\prime \prime} a_{1}^{\prime \prime} \ldots \delta^{\prime \prime} a_{n}^{\prime \prime}$ :

$$
\begin{aligned}
\bar{i} \circ \bar{j}^{\prime \prime}\left(\omega^{\prime \prime}\right) & =\bar{i}\left[\left(\mathbf{1} \hat{\otimes} a_{0}^{\prime \prime}\right) \delta\left(\mathbf{1} \hat{\otimes} a_{1}^{\prime \prime}\right) \ldots \delta\left(\mathbf{1} \hat{\otimes} a_{n}^{\prime \prime}\right)\right. \\
& =\left(\mathbf{1} \hat{\otimes} a_{0}^{\prime \prime}\right)\left(\mathbf{1} \hat{\otimes} \delta^{\prime \prime} a_{1}^{\prime \prime}\right) \ldots\left(\mathbf{1} \hat{\otimes} \delta^{\prime \prime} a_{n}^{\prime \prime}\right)=\mathbf{1} \hat{\otimes} \omega^{\prime \prime} .
\end{aligned}
$$

Check of $\bar{i} \circ J=\operatorname{id}_{\Omega \mathbf{A}^{\prime}} \hat{\otimes} \Omega \mathbf{A}^{\prime \prime}$ : we have, owing to (9):

$$
\begin{aligned}
\bar{i} \circ J\left(\omega^{\prime} \hat{\otimes} \omega^{\prime \prime}\right) & =\bar{i}\left[\bar{j}^{\prime}\left(\omega^{\prime}\right) \bar{j}^{\prime \prime}\left(\omega^{\prime \prime}\right)\right]=\bar{i} \circ \bar{j}^{\prime}\left(\omega^{\prime}\right) \cdot \bar{i} \circ \bar{j}^{\prime \prime}\left(\omega^{\prime \prime}\right) \\
& =\left(\omega^{\prime} \hat{\otimes} \mathbf{1}\right)\left(\mathbf{1} \hat{\otimes} \omega^{\prime \prime}\right)=\omega^{\prime} \hat{\otimes} \omega^{\prime \prime} .
\end{aligned}
$$

\section{Study of $\pi_{D}(\Omega \mathrm{A})$}

II.0 Assumptions and Notation. In what follows we give ourselves two $\mathbf{Z} / 2$-graded unital real (or complex) *-algebras $\mathbf{A}^{\prime}=\mathbf{A}^{\prime 0} \oplus \mathbf{A}^{\prime 1}$ and $\mathbf{A}^{\prime \prime}=\mathbf{A}^{\prime \prime 0} \oplus \mathbf{A}^{\prime \prime 1}$ with respective grading involutions $\theta^{\prime}$ and $\theta^{\prime \prime}$, and respective unital differential envelopes $\Omega \mathbf{A}^{\prime}$ and $\Omega \mathbf{A}^{\prime \prime} . \mathbf{A}^{\prime}$ and $\mathbf{A}^{\prime \prime}$ are equipped with respective even $K$-cycles $\left(H^{\prime}, D^{\prime}, \chi^{\prime}\right)$ and $\left(H^{\prime \prime}, D^{\prime \prime}, \chi^{\prime \prime}\right){ }^{4}$ We consider the skew tensor-product

$$
\mathbf{A}=\mathbf{A}^{\prime} \hat{\otimes} \mathbf{A}^{\prime \prime}
$$

${ }_{4}$ A $K$-cycle $(H, D, \chi)$ of a $\mathbf{Z} / 2$-graded algebra $A=A^{0} \oplus A^{1}$ is a $\mathbf{Z} / 2$-graded Hilbert space $H=H^{0} \oplus H^{1}$ with graded involution $\chi$ carrying a grade-preserving $*$-representation $\pi$ of $A$ into $\left(B(H) ; \mathrm{ad}_{\chi}\right)$, and an odd self-adjoint operator $D$ such that all graded commutators $[D, \pi(a)]$, $a \in A$, are compact. Practically we are interested in the case where $\mathbf{A}^{\prime}$ is equipped with an even $d^{+}$-summable $K$-cycle $\left(H^{\prime}, D^{\prime}, \chi^{\prime}\right), d^{\prime}=2 m^{\prime}, m^{\prime} \in \mathbf{N}$, and $\mathbf{A}^{\prime \prime}$ has finite dimension and is equipped with an even $K$-cycle $\left(H^{\prime \prime}, D^{\prime \prime}, \chi^{\prime \prime}\right)$ for which the Hilbert space $H^{\prime \prime}$ is finite-dimensional 
equipped with the (skew) tensor-product $K$-cycle

$$
(H, D, \chi)=\left(H^{\prime}, D^{\prime}, \chi^{\prime}\right) \hat{\otimes}\left(H^{\prime \prime}, D^{\prime \prime}, \chi^{\prime \prime}\right) .
$$

We recall that the skew tensor-product $\mathbf{A}^{\prime} \hat{\otimes} \mathbf{A}^{\prime \prime}$ is the $\mathbf{Z} / 2$-graded algebra obtained by equipping the vector space $\mathbf{A}^{\prime} \otimes \mathbf{A}^{\prime \prime}$ (algebraic skew tensor product) with the grading $\theta^{\prime} \otimes \theta^{\prime \prime}$, and the (associative) algebraic product: ${ }^{5}$

$$
\begin{aligned}
& \left(a^{\prime} \hat{\otimes} a^{\prime \prime}\right)\left(b^{\prime} \hat{\otimes} b^{\prime \prime}\right) \\
& \quad=(-1)^{\partial a^{\prime \prime} \cdot \partial b^{\prime}}\left(a^{\prime} b^{\prime} \hat{\otimes} a^{\prime \prime} b^{\prime \prime}\right), \quad a^{\prime}, b^{\prime} \in \mathbf{A}^{\prime 0} \cup \mathbf{A}^{\prime \prime}, a^{\prime \prime}, b^{\prime \prime} \in \mathbf{A}^{\prime \prime 0} \cup \mathbf{A}^{\prime \prime} .
\end{aligned}
$$

We recall the definition of the (skew) tensor-product $K$-cycle: with the symbols $\otimes$, resp. $\hat{\otimes}$, indicating ordinary, resp. skew tensor-products, (over $\mathbf{R}$ (or $\mathbf{C}$ )) one has: ${ }^{6}$

$$
\left\{\begin{array}{l}
H=H^{\prime} \otimes H^{\prime \prime}, \\
\pi=\pi^{\prime} \hat{\otimes} \pi^{\prime \prime}, \text { i.e. } \pi\left(a^{\prime} \hat{\otimes} a^{\prime \prime}\right)=\pi^{\prime}\left(a^{\prime}\right) \chi^{\prime \partial a^{\prime \prime}} \otimes \pi^{\prime \prime}\left(a^{\prime \prime}\right), \\
\quad a^{\prime} \in \mathbf{A}^{\prime}, a^{\prime \prime} \in \mathbf{A}^{\prime \prime 0} \cup \mathbf{A}^{\prime \prime 1}, \\
D=D^{\prime} \hat{\otimes} \mathbf{1}_{H^{\prime \prime}}+\mathbf{1}_{H^{\prime}} \hat{\otimes} D^{\prime \prime}=D^{\prime} \hat{\otimes} \mathbf{1}_{H^{\prime \prime}}+\chi^{\prime} \otimes D^{\prime \prime}, \\
\chi=\chi^{\prime} \otimes \chi^{\prime \prime} .
\end{array}\right.
$$

We shall write in the sequel $\pi_{D}=\pi, \pi_{D^{\prime}}=\pi^{\prime}$ and $\pi_{D^{\prime \prime}}=\pi^{\prime \prime}$ for the respective representations of the *-algebras $\Omega \mathbf{A}, \Omega \mathbf{A}^{\prime}$ and $\Omega \mathbf{A}^{\prime \prime}$ induced by $D, D^{\prime}$ and $D^{\prime \prime}$ on $H, H^{\prime}$ and $H^{\prime \prime}$. We recall, with $[.,$.$] denoting the graded commutator, and$ $a_{0}, a_{1}, \ldots, a_{n} \in \mathbf{A}, a_{0}^{\prime}, a_{1}^{\prime}, \ldots, a_{n}^{\prime} \in \mathbf{A}^{\prime}, a_{0}^{\prime \prime}, a_{1}^{\prime \prime}, \ldots, a_{n}^{\prime \prime} \in \mathbf{A}^{\prime \prime}$, that one has:

$$
\left\{\begin{array}{l}
\pi\left(a_{0} \delta a_{1} \ldots \delta a_{n}\right) \\
\quad=(-i)^{n} \pi\left(a_{0}\right)\left[D, \pi\left(a_{1}\right)\right]\left[D, \pi\left(a_{2}\right)\right] \ldots\left[D, \pi\left(a_{n}\right)\right] \\
\pi^{\prime}\left(a_{0}^{\prime} \delta^{\prime} a_{1}^{\prime} \ldots \delta^{\prime} a_{n}^{\prime}\right) \\
\quad=(-i)^{n} \pi^{\prime}\left(a_{0}^{\prime}\right)\left[D^{\prime}, \pi^{\prime}\left(a_{1}^{\prime}\right)\right]\left[D^{\prime}, \pi^{\prime}\left(a_{2}^{\prime}\right)\right] \ldots\left[D^{\prime}, \pi^{\prime}\left(a_{n}^{\prime}\right)\right] \\
\pi^{\prime \prime}\left(a_{0}^{\prime \prime} \delta^{\prime \prime} a_{1}^{\prime \prime} \ldots \delta^{\prime \prime} a_{n}^{\prime \prime}\right) \\
\quad=(-i)^{n} \pi^{\prime \prime}\left(a_{0}^{\prime \prime}\right)\left[D^{\prime \prime}, \pi^{\prime \prime}\left(a_{1}^{\prime \prime}\right)\right]\left[D^{\prime \prime}, \pi^{\prime \prime}\left(a_{2}^{\prime \prime}\right)\right] \ldots\left[D^{\prime \prime}, \pi^{\prime \prime}\left(a_{n}^{\prime \prime}\right)\right] .
\end{array}\right.
$$

II.1 Lemma. (i) The representation $\pi$ of $\Omega \mathbf{A}$ factors through the homomorphism $\bar{i}$ : one has:

$$
\pi=\pi_{\otimes} \circ \bar{i}
$$

with $\pi_{\otimes}$ the representation of $\Omega_{\otimes} \mathbf{A}$ given by:

$$
\begin{aligned}
& \pi_{\otimes} {\left[\left(a_{0}^{\prime} \otimes a_{0}^{\prime \prime}\right) \delta_{\otimes}\left(a_{1}^{\prime} \otimes a_{1}^{\prime \prime}\right) \ldots \delta_{\otimes}\left(a_{n}^{\prime} \otimes a_{n}^{\prime \prime}\right)\right] } \\
& \quad=(-i)^{n} \pi\left(a_{0}^{\prime} \otimes a_{0}^{\prime \prime}\right)\left[D, \pi\left(a_{1}^{\prime} \otimes a_{1}^{\prime \prime}\right)\right] \ldots\left[D, \pi\left(a_{n}^{\prime} \otimes a_{n}^{\prime \prime}\right)\right] .
\end{aligned}
$$

(ii) One has, with $\mathbf{K}=\operatorname{Ker} \pi$ and $\mathbf{K}_{\otimes}=\operatorname{Ker} \pi_{\otimes}$ :

$$
\mathbf{K}=\bar{i}^{-1}\left(\mathbf{K}_{\otimes}\right)
$$

\footnotetext{
5 As above, one has $a^{\prime} \hat{\otimes} a^{\prime \prime}=a^{\prime} \otimes a^{\prime \prime}$, the symbol $\hat{\otimes}$ reminding us of the multiplication rule (15) ${ }^{6}$ Let $H$ be a $\mathbf{Z} / 2$-graded vector space with grading involution $\chi$ : we recall that End $H$ is canonically a $\mathbf{Z} / 2$-graded algebra under the grading involution $\mathrm{ad}_{\chi}$. Let $H^{\prime}$ and $H^{\prime \prime}$ be $\mathbf{Z} / 2$-graded vector spaces with respective grading involutions $\chi^{\prime}$ and $\chi^{\prime \prime}$. We recall that one then gets a homomorphism of Z/2-graded algebras: End $H^{\prime} \hat{\otimes}$ End $H^{\prime \prime} \rightarrow \operatorname{End}\left(H^{\prime} \otimes H^{\prime \prime}\right)$ by representating the skew tensor product End $H^{\prime} \hat{\otimes}$ End $H^{\prime \prime}$ as follows: $A^{\prime} \hat{\otimes} A^{\prime \prime} \rightarrow A^{\prime} \chi^{\prime \partial A^{\prime \prime}} \otimes A^{\prime \prime}, A^{\prime} \in$ End $H^{\prime}, A^{\prime \prime} \in$ End $H^{\prime \prime}$
} 
from which follows by surjectivity of $\bar{i}$ :

$$
\mathbf{K}_{\otimes}=\bar{i}(\mathbf{K}) .
$$

(iii) One has:

$$
\left\{\begin{array}{l}
\pi\left(\Omega \mathbf{A}^{n}\right)=\pi_{\otimes}\left(\Omega_{\otimes} \mathbf{A}^{n}\right) \\
\pi\left(\delta \mathbf{K}^{n-1}\right)=\pi_{\otimes}\left(\delta_{\otimes} \mathbf{K}_{\otimes}^{n-1}\right) .
\end{array}\right.
$$

Proof. (i) $\pi_{\otimes}$ as given by (19) is a representation of $\Omega_{\otimes} \mathbf{A}$ because of the graded derivation property of $[D,$.$] .$

Check of (18): With $\omega=\left(a_{0}^{\prime} \otimes a_{0}^{\prime \prime}\right) \delta\left(a_{1}^{\prime} \otimes a_{1}^{\prime \prime}\right) \ldots \delta\left(a_{n}^{\prime} \otimes a_{n}^{\prime \prime}\right) \in \Omega$ A the left-hand side of (19) is $\pi_{\otimes} \circ \bar{i}(\omega)$ whilst its right-hand side equals $\pi(\omega)$. (ii) follows from the equivalences $\omega \in \mathbf{K} \Leftrightarrow \pi(\omega)=0 \Leftrightarrow \pi_{\otimes} \circ \bar{i}(\omega)=0 \Leftrightarrow \bar{i}(\omega) \in \mathbf{K}_{\otimes}$. (iii) The first line in (21) is obvious. For the second assertion, we have by (5), (18) and (20a):

$$
\begin{aligned}
\pi\left(\delta \mathbf{K}^{n-1}\right) & =\left(\pi_{\otimes} \circ \bar{i} \circ \delta\right)\left(\mathbf{K}^{n-1}\right) \\
& =\left(\pi_{\otimes} \circ \delta_{\otimes} \circ \bar{i}\right)\left(\mathbf{K}^{n-1}\right)=\pi_{\otimes}\left(\delta_{\otimes} \mathbf{K}_{\otimes}^{n-1}\right) .
\end{aligned}
$$

II.2 Lemma. (i) With the assumptions II.0 we have that the representation $\pi_{\otimes}$ is the skew tensor product of the representations $\pi^{\prime}$ and $\pi^{\prime \prime}$ in the following sense: one has, for $\omega^{\prime} \in \Omega \mathbf{A}^{\prime p}, \omega^{\prime \prime} \in \Omega \mathbf{A}^{\prime \prime q}$ :

$$
\begin{aligned}
\pi_{\otimes}\left(\omega^{\prime} \hat{\otimes} \omega^{\prime \prime}\right) & =\pi^{\prime}\left(\omega^{\prime}\right) \hat{\otimes} \pi^{\prime \prime}\left(\omega^{\prime \prime}\right) \\
& =\pi^{\prime}\left(\omega^{\prime}\right) \chi^{q} \otimes \pi^{\prime \prime}\left(\omega^{\prime \prime}\right)= \begin{cases}\pi^{\prime}\left(\omega^{\prime}\right) \otimes \pi^{\prime \prime}\left(\omega^{\prime \prime}\right), & q \text { even }, \\
\pi^{\prime}\left(\omega^{\prime}\right) \chi^{\prime} \otimes \pi^{\prime \prime}\left(\omega^{\prime \prime}\right), & \text { q odd },\end{cases}
\end{aligned}
$$

where $\otimes$ denotes ordinary, and $\hat{\otimes}$ skew tensor products. In other terms one has a homomorphism $\pi_{\otimes}: \Omega \mathbf{A}^{\prime} \hat{\otimes} \Omega \mathbf{A}^{\prime \prime} \rightarrow$ End $H^{\prime} \hat{\otimes}$ End $H^{\prime \prime}$ of skew tensor products of $\mathbf{Z} / 2$ graded algebras ${ }^{6}$

(ii) One has accordingly:

$$
\pi\left(\Omega \mathbf{A}^{n}\right)=\pi_{\otimes}\left(\Omega_{\otimes} \mathbf{A}^{n}\right)=\sum_{p+q=n} \pi^{\prime}\left(\Omega \mathbf{A}^{\prime p}\right) \hat{\otimes} \pi^{\prime \prime}\left(\Omega \mathbf{A}^{\prime \prime q}\right)
$$

Proof. It is enough to check (23) for $\omega^{\prime}=a_{0}^{\prime} \delta^{\prime} a_{1}^{\prime} \ldots \delta^{\prime} a_{p}^{\prime}$ and $\omega^{\prime \prime}=a_{0}^{\prime \prime} \delta^{\prime \prime} a_{1}^{\prime \prime} \ldots \delta^{\prime \prime} a_{q}^{\prime \prime}$ with $a_{1}^{\prime}, \ldots, a_{p}^{\prime} \in \mathbf{A}^{\prime 0} \cup \mathbf{A}^{\prime 1}$ and $a_{0}^{\prime \prime}, a_{1}^{\prime \prime}, \ldots, a_{q}^{\prime \prime} \in \mathbf{A}^{\prime \prime 0} \cup \mathbf{A}^{\prime \prime 1}, p, q \in \mathbf{N}$. However, since the product $\hat{\otimes}$ is associative, and we have $\omega^{\prime} \hat{\otimes} \omega^{\prime \prime}=\left(\omega^{\prime} \hat{\otimes} \mathbf{1}\right)\left(\mathbf{1} \hat{\otimes} \omega^{\prime \prime}\right)$ with

$$
\left\{\begin{array}{l}
\omega^{\prime} \hat{\otimes} \mathbf{1}=\left(a_{0}^{\prime} \hat{\otimes} \mathbf{1}\right)\left(\delta^{\prime} a_{1}^{\prime} \hat{\otimes} \mathbf{1}\right) \ldots\left(\delta^{\prime} a_{p}^{\prime} \hat{\otimes} \mathbf{1}\right) \\
\mathbf{1} \hat{\otimes} \omega^{\prime \prime}=\left(\mathbf{1} \hat{\otimes} a_{0}^{\prime \prime}\right)\left(\mathbf{1} \hat{\otimes} \delta^{\prime \prime} a_{1}^{\prime \prime}\right) \ldots\left(\mathbf{1} \hat{\otimes} \delta^{\prime \prime} a_{1}^{\prime \prime}\right),
\end{array}\right.
$$

it suffices to check (23) for each type of factors occurring in (25). For the types $\left(a_{0}^{\prime} \hat{\otimes} \mathbf{1}\right)$ and $\left(\mathbf{1} \hat{\otimes} a_{0}^{\prime \prime}\right)$, this follows from the second line (16). For the type $\mathbf{1} \hat{\otimes} \delta^{\prime \prime} a^{\prime \prime}$, $a^{\prime \prime} \in \mathbf{A}^{\prime \prime 0} \cup \mathbf{A}^{\prime \prime 1}$, we have by I and by the definitions of $\pi_{\otimes}$ and $D$ :

$$
\begin{aligned}
\pi_{\otimes}\left(\mathbf{1} \hat{\otimes} \delta^{\prime \prime} a^{\prime \prime}\right)= & -i\left[D, \pi\left(\mathbf{1} \hat{\otimes} a^{\prime \prime}\right)\right]=-i\left[D^{\prime} \otimes \mathbf{1}+\chi^{\prime} \otimes D^{\prime \prime}, \mathbf{1} \otimes \pi^{\prime \prime}\left(a^{\prime \prime}\right)\right] \\
= & -i\left\{D^{\prime} \otimes \pi^{\prime \prime}\left(a^{\prime \prime}\right)+\chi^{\prime} \otimes D^{\prime \prime} \pi^{\prime \prime}\left(a^{\prime \prime}\right)\right. \\
& \left.-(-1)^{\partial a^{\prime \prime}}\left\{(-1)^{\partial a^{\prime \prime}} D^{\prime} \otimes \pi^{\prime \prime}\left(a^{\prime \prime}\right)+\chi^{\prime} \otimes \pi^{\prime \prime}\left(a^{\prime \prime}\right) D^{\prime \prime}\right\}\right\} \\
= & \left.-i\left\{\chi^{\prime} \otimes D^{\prime \prime} \pi^{\prime \prime}\left(a^{\prime \prime}\right)-(-1)^{\partial a^{\prime \prime}} \chi^{\prime} \otimes \pi^{\prime \prime}\left(a^{\prime \prime}\right) D^{\prime \prime}\right)\right\} \\
= & -i\left\{\chi^{\prime} \otimes\left[D^{\prime \prime}, \pi^{\prime \prime}\left(a^{\prime \prime}\right)\right]\right\}=\chi^{\prime} \otimes \pi^{\prime \prime}\left(\delta^{\prime \prime} a^{\prime \prime}\right) .
\end{aligned}
$$


We have, analogously, for the type $\delta^{\prime} a^{\prime} \hat{\otimes} \mathbf{1}, a^{\prime} \in \mathbf{A}^{\prime 0} \cup \mathbf{A}^{\prime 1}$ :

$$
\begin{aligned}
\pi_{\otimes}\left(\delta^{\prime} a^{\prime} \hat{\otimes} \mathbf{1}\right)= & -i\left[D, \pi\left(a^{\prime} \otimes \mathbf{1}\right)\right]=-i\left[D^{\prime} \otimes \mathbf{1}+\chi^{\prime} \otimes D^{\prime \prime}, \pi^{\prime}\left(a^{\prime}\right) \otimes \mathbf{1}\right] \\
= & -i\left\{D^{\prime} \pi^{\prime}\left(a^{\prime}\right) \otimes \mathbf{1}+(-1)^{\partial a^{\prime}} \chi^{\prime} \pi^{\prime}\left(a^{\prime}\right) \otimes D^{\prime \prime}\right. \\
& -(-1)^{\partial a^{\prime}}\left\{\pi^{\prime}\left(a^{\prime}\right) D^{\prime} \otimes \mathbf{1}+\pi^{\prime}\left(a^{\prime}\right) \chi^{\prime} \otimes D^{\prime \prime}\right\} \\
= & \left.-i\left\{\left[\chi^{\prime}, \pi^{\prime}\left(a^{\prime}\right)\right] \otimes D^{\prime \prime}+\left[D^{\prime}, \pi^{\prime}\left(a^{\prime}\right)\right]\right\} \otimes \mathbf{1}\right\} \\
= & -i\left[D^{\prime}, \pi^{\prime}\left(a^{\prime}\right)\right] \otimes \mathbf{1} \\
= & \pi^{\prime}\left(\delta^{\prime} a^{\prime}\right) \otimes \mathbf{1} .
\end{aligned}
$$

As a consequence of what precedes, we now describe a result useful for the computation of the module $\Omega_{D} \mathbf{A}^{2}$ of quantum two-forms in the tensor-product case, which we recall to be given by [2]:

$$
\Omega_{D} \mathbf{A}^{2}=\frac{\pi_{D}\left(\Omega \mathbf{A}^{2}\right)}{\pi_{D}\left(\delta K^{1}\right)} .
$$

We have:

II.3 Proposition. Let $\mathbf{A}=\mathbf{A}^{\prime} \hat{\otimes} \mathbf{A}^{\prime \prime},\left(H^{\prime}, D^{\prime}, \chi^{\prime}\right),\left(H^{\prime \prime}, D^{\prime \prime}, \chi^{\prime \prime}\right), \pi, \pi^{\prime}$, and $\pi^{\prime \prime}$ be as in II.0 and assume in addition $\pi^{\prime}$ and $\pi^{\prime \prime}$ faithful. We have tensorial decompositions:

$$
\pi\left(\Omega \mathbf{A}^{1}\right)=\pi^{\prime}\left(\Omega \mathbf{A}^{\prime 1}\right) \hat{\otimes} \pi^{\prime \prime}\left(\mathbf{A}^{\prime \prime}\right) \oplus \pi^{\prime}\left(\mathbf{A}^{\prime}\right) \hat{\otimes} \pi^{\prime \prime}\left(\Omega \mathbf{A}^{\prime \prime 1}\right),
$$

i.e.

$$
\Omega_{D} \mathbf{A}^{1}=\left(\Omega_{D^{\prime}} \mathbf{A}^{\prime 1} \hat{\otimes} \mathbf{A}^{\prime \prime}\right) \oplus\left(\mathbf{A}^{\prime} \hat{\otimes} \Omega_{D^{\prime \prime}} \mathbf{A}^{\prime \prime 1}\right)
$$

further

$$
\pi\left(\Omega \mathbf{A}^{2}\right)=\pi^{\prime}\left(\Omega \mathbf{A}^{\prime 2}\right) \hat{\otimes} \pi^{\prime \prime}\left(\mathbf{A}^{\prime \prime}\right)+\pi^{\prime}\left(\Omega \mathbf{A}^{\prime 1}\right) \hat{\otimes} \pi\left(\Omega \mathbf{A}^{\prime \prime 1}\right)+\pi^{\prime}\left(\mathbf{A}^{\prime}\right) \hat{\otimes} \pi^{\prime \prime}\left(\Omega \mathbf{A}^{\prime \prime 2}\right),
$$

and, for the respective kernels $K_{\otimes}, K^{\prime}, K^{\prime \prime}$ of $\pi_{\otimes}, \pi^{\prime}$, and $\pi^{\prime \prime}$ :

$$
\begin{gathered}
K_{\otimes}^{1}=K^{\prime 1} \hat{\otimes} \mathbf{A}^{\prime \prime}+\mathbf{A}^{\prime} \hat{\otimes} K^{\prime \prime 1}, \\
\pi\left(\delta K^{1}\right)=\pi^{\prime}\left(\delta^{\prime} K^{\prime 1}\right) \hat{\otimes} \pi^{\prime \prime}\left(\mathbf{A}^{\prime \prime}\right)+\pi^{\prime}\left(\mathbf{A}^{\prime}\right) \hat{\otimes} \pi^{\prime \prime}\left(\delta^{\prime \prime} K^{\prime \prime 1}\right) .
\end{gathered}
$$

Proof. Equations (29) and (30) are special cases of (24) and (29a) follows from the faithfulness of the representations $\pi^{\prime}$, and $\pi^{\prime \prime}$. Proof of (31): the inclusion $\supset$ is obvious from (23). Proof of the reverse inclusion: by (29) the most general element of $\Omega_{\otimes} \mathbf{A}^{1}$ is of the type $\omega=\sum_{\imath=1, \ldots, n} \omega_{1}^{\prime} \otimes f_{i}^{\prime \prime}+\sum_{\imath=1, \ldots, n} f_{\imath}^{\prime} \otimes \omega_{\imath}^{\prime \prime}, \omega_{\imath}^{\prime} \in \Omega \mathbf{A}^{\prime 1}, f_{i}^{\prime \prime} \in \mathbf{A}^{\prime \prime}, f_{\imath}^{\prime} \in \mathbf{A}^{\prime}$, $\omega_{\imath}^{\prime \prime} \in \Omega \mathbf{A}^{\prime \prime \prime}$, with the $f_{\imath}^{\prime}$, resp. the $f_{i}^{\prime \prime}$, linearly independent: by (23) $\omega$ belongs to $K_{\otimes}^{1}$ iff one has:

$$
0=\sum_{\imath=1, \ldots, n} \pi^{\prime}\left(\omega_{\imath}^{\prime}\right) \otimes \pi^{\prime \prime}\left(f_{\imath}^{\prime \prime}\right)+\sum_{\imath=1, \ldots, n} \pi^{\prime}\left(f_{\imath}^{\prime}\right) \chi^{\prime} \otimes \pi^{\prime \prime}\left(\omega_{\imath}^{\prime \prime}\right)
$$

which ${ }^{7}$ amounts to

$$
0=\sum_{\imath=1, \ldots, n} \pi^{\prime}\left(\omega_{1}^{\prime}\right) \otimes \pi^{\prime \prime}\left(f_{i}^{\prime \prime}\right)=\sum_{\imath=1, \ldots, n} \pi^{\prime}\left(f_{i}^{\prime}\right) \otimes \pi^{\prime \prime}\left(\omega_{\imath}^{\prime \prime}\right)
$$

\footnotetext{
7 In fact intertwining $\theta^{\prime} \otimes$ id with ad $\chi^{\prime}$ and id $\otimes \theta^{\prime \prime}$ with ad $\chi^{\prime \prime}$, where $\theta^{\prime}$ and $\theta^{\prime \prime}$ are the respective total-grading involutions of $\Omega \mathbf{A}^{\prime}$ and $\Omega \mathbf{A}^{\prime \prime}$ : one has $\pi\left[\left(\theta^{\prime} \otimes \mathrm{id}\right)\left(\omega^{\prime} \otimes \mathbf{1}\right)\right]=\left(\chi^{\prime} \otimes \mathbf{1}\right) \pi\left(\omega^{\prime} \otimes \mathbf{1}\right)\left(\chi^{\prime} \otimes \mathbf{1}\right)$, $\omega^{\prime} \in \Omega \mathbf{A}^{\prime} ;$ and $\pi\left[\left(\mathrm{id} \otimes \theta^{\prime \prime}\right)\left(\mathbf{1} \otimes \omega^{\prime \prime}\right)\right]=\left(\mathbf{1} \otimes \chi^{\prime \prime}\right) \pi\left(\mathbf{1} \otimes \omega^{\prime \prime}\right)\left(\mathbf{1} \otimes \chi^{\prime \prime}\right), \omega^{\prime \prime} \in \Omega \mathbf{A}^{\prime \prime}$
} 
To conclude from this the vanishing of all $\pi^{\prime}\left(\omega_{\imath}^{\prime}\right)$ and all $\pi^{\prime \prime}\left(\omega_{i}^{\prime \prime}\right)$, we notice that, by the faithfulness of $\pi^{\prime}$ and $\pi^{\prime \prime}$, the $\pi^{\prime}\left(f_{\imath}^{\prime}\right)$, resp. the $\pi^{\prime \prime}\left(f_{\imath}^{\prime \prime}\right)$, are linearly independent, and we invoke the following result, special case of the statement in Exercise 6, Chapitre 1, Paragraphe 2, of [5]: given two Hilbert spaces $H$ and $H^{\prime}$, and operators $T_{i} \in B(H)$, $T_{\imath}^{\prime} \in B\left(H^{\prime}\right), i=1, \ldots, n$, such that the $T_{i}$ are linearly independent and such that $\sum_{i=1, \ldots, n} T_{i} \otimes T_{i}^{\prime}=0$, it follows that all $T_{\imath}^{\prime}$ vanish: we proved (31) from which (32) now follows by the fact that

$$
\begin{aligned}
& \delta\left(\sum_{i=1, \ldots, n} \omega_{\imath}^{\prime} \otimes f_{\imath}^{\prime \prime}+\sum_{\imath=1, \ldots, n} f_{i}^{\prime} \otimes \omega_{i}^{\prime \prime}\right) \\
& =\sum_{i=1, \ldots, n}\left[\delta^{\prime} \omega_{i}^{\prime} \otimes f_{\imath}^{\prime \prime}-\omega_{\imath}^{\prime} \otimes \delta^{\prime \prime} f_{i}^{\prime \prime}+\delta^{\prime} f_{i}^{\prime} \otimes \omega_{i}^{\prime \prime}+f_{i}^{\prime} \otimes \delta^{\prime \prime} \omega_{i}^{\prime \prime}\right]
\end{aligned}
$$

whence, for $\omega_{i}^{\prime} \in K^{\prime 1}$ and $\omega_{i}^{\prime \prime} \in K^{\prime \prime 1}$ :

$$
\begin{aligned}
& \pi\left[\delta\left(\sum_{i=1, \ldots, n} \omega_{i}^{\prime} \otimes f_{\imath}^{\prime \prime}+\sum_{\imath=1, \ldots, n} f_{\imath}^{\prime} \otimes \omega_{i}^{\prime \prime}\right)\right] \\
& \quad=\sum_{i=1, \ldots, n}\left[\pi^{\prime}\left(\delta^{\prime} \omega_{i}^{\prime}\right) \otimes \pi^{\prime \prime}\left(f_{i}^{\prime \prime}\right)+\pi^{\prime}\left(f_{\imath}^{\prime}\right) \otimes \pi^{\prime \prime}\left(\delta^{\prime \prime} \omega_{\imath}^{\prime \prime}\right)\right] .
\end{aligned}
$$

Acknowledgements. We are grateful to the referee for pointing out an error, to Alain Connes for providing a counterexample, and to Bruno Iochum and Peter Seibt for discussions.

\section{References}

1. Connes, A., Lott, J.: Particle models and non-commutative geometry. Nucl. Phys. B 18 (Proc. Suppl.) (1990)

2. Connes, A.: The metric aspects of non-commutative geometry. Collège de France Preprint (1991)

3. Kastler, D.: A detailed account of Alain Connes' version of the standard model in noncommutative geometry. I CPT-Marseille Preprint (1991) revised (1992) and III (1992)

4. Kastler, D.: Cyclic cohomology within the differential envelope. Travaux en cours, 30, Paris: Hermann 1988

5. Dixmier, J.: Les algèbres d'opérateurs dans l'espace Hilbertien. Cahiers scientifiques XXV Paris: Gauthiers-Villars 1969

Communicated by A. Jaffe 